\title{
ON THE COMPLEX BORDISM AND COBORDISM OF INFINITE COMPLEXES
}

\author{
BY PETER S. LANDWEBER ${ }^{1}$
}

Communicated by Raoul Bott, November 20, 1969

Let $M U_{*}()$ and $M U^{*}()$ denote the reduced homology (complex bordism) and cohomology (complex cobordism) functors represented by the unitary Thom spectrum $M U$. Two examples of the immense richness of these functors are provided by the development (for suitable complexes or spectra) of the Adams spectral sequence

$$
\operatorname{Ext}_{M U^{*}(M U)}^{*, *}\left(M U^{*}(X), M U^{*}(Y)\right) \Rightarrow\{Y, X\}_{*}
$$

by S. P. Novikov [12], and the universal coefficient theorem

$$
\operatorname{Tor}_{*, *}^{M U^{*}\left(S^{0}\right)}\left(M U_{*}(X), \boldsymbol{Z}\right) \Rightarrow H_{*}(X ; \boldsymbol{Z})
$$

by P. E. Conner and L. Smith [9]. Recently N. A. Baas [4] has written an excellent account of the Adams spectral sequence (1), and J. F. Adams has made a thorough analysis of universal coefficient theorems such as (2) in Lecture 1 of [1].

In $\$ 1$ we announce several solutions to the problem-when is $M U^{*}(X)$ isomorphic to the inverse limit of the complex cobordism of the skeleta (assumed finite) of $X$ ? In the remaining sections we illustrate several universal coefficient theorems, among them (2), by announcing the results of several computations for EilenbergMacLane spectra $K(\pi)$ and the spectrum $b u$ which represents connective $K$-theory. Full details will appear elsewhere.

1. Let $X$ denote a based CW-complex or highly connected CWspectrum as defined by J. M. Boardman [5]. We shall assume that each skeleton $X^{\mu}$ of $X$ is a finite complex, and define a filtration of $M U^{t}(X)$ by the subgroups $M U_{\mu}^{t}(X)=\operatorname{Ker}\left\{M U^{t}(X) \rightarrow M U^{t}\left(X^{\mu-1}\right)\right\}$. In [4] Baas emphasizes the importance, for the construction of the Adams spectral sequence (1), of dealing with spectra for which the filtration topology on the cobordism groups $M U^{t}(X)$ is complete and Hausdorff. In [6] and [7] V. M. Buhštaber and A. S. Miščenko

AMS Subject Classifications. Primary 5530; Secondary 5710.

Key Words and Phrases. Complex bordism, complex cobordism, universal coefficient theorems, Eilenberg-MacLane spectra.

${ }^{1}$ Research supported in part by NSF grant GP-7993. 
have studied from several points of view the condition that $K^{*}(X)$ be complete and Hausdorff. Their methods are applicable to complex cobordism with only minor modifications, and lead to the following three results.

TheOREM 1. $M U^{t}(X)$ is complete and Hausdorff for all $t$ if and only if the homomorphism $\mu \otimes Q: M U^{*}(X) \otimes Q \rightarrow H^{*}(X ; Q)$ induced by the Thom homomorphism $\mu: M U^{*}(X) \rightarrow H^{*}(X ; \boldsymbol{Z})$ is an epimorphism.

THEOREM 2. $M U^{t+1}(X)$ is complete and Hausdorff if and only if the inverse system $\left\{M U^{t}\left(X^{\mu}\right)\right\}$ satisfies the Mittag-Leffler condition $($ see $[3, \S 3])$. In this case the following stronger condition holds: for each $\mu$ there exists $\nu \geqq \mu$ such that the image of $M U^{t}(X)$ in $M U^{t}\left(X^{\mu}\right)$ under restriction coincides with the image of $M U^{t}\left(X^{\nu}\right)$ in $M U^{t}\left(X^{\mu}\right)$.

THEOREM 3. $M U^{t}(X)$ is complete and Hausdorff for all $t$ if and only if, in the Atiyah-Hirzebruch spectral sequence $\left\{E_{r}, d_{r}\right\}$ associated to the skeleton filtration of $X$ for the cohomology theory $M U^{*}(), E_{r}^{p, q}=E_{\infty}^{p, q}$ for $r$ sufficiently large depending on $p$ and $q$. In this case, the spectral sequence is strongly convergent as well.

The following result (compare $[4, \S 5 . \mathrm{A}]$ ) is a preliminary to the proofs of these theorems, and is read off directly from the exact sequence (Milnor's lemma [11])

(3) $0 \rightarrow \operatorname{proj} \lim ^{1} M U^{t-1}\left(X^{\mu}\right) \rightarrow M U^{t}(X) \rightarrow \operatorname{proj} \lim M U^{t}\left(X^{\mu}\right) \rightarrow 0$.

For later use put proj $M U^{t}(X)=\operatorname{proj} \lim M U^{t}\left(X^{\mu}\right)$.

Proposition 4. The following properties are equivalent:

(a) $M U^{t}(X)$ is complete and Hausdorf,

(b) $M U^{t}(X)$ is Hausdorff,

(c) $\cap_{\mu} M U_{\mu}^{\imath}(X)=0$,

(d) proj $\lim ^{1} M U^{t-1}\left(X^{\mu}\right)=0$, and

(e) the natural map $M U^{t}(X) \rightarrow \operatorname{proj} M U^{t}(X)$ is an isomorphism.

REMARK. We regard Theorem 2 as the main technical result, since its proof leads one to a better setting for the ingenious argument used by Buhštaber and Miščenko to prove the analogue in $K$-theory of Theorem 1. If $M U^{t+1}(X)$ is Hausdorff, then one can prove rather directly that

$$
\operatorname{Im}\left\{M U^{t}(X) \rightarrow M U^{t}\left(X^{\mu}\right)\right\}=\bigcap_{\nu \geq \mu} \operatorname{Im}\left\{M U^{t}\left(X^{\nu}\right) \rightarrow M U^{t}\left(X^{\mu}\right)\right\}
$$

(the analogue of strong convergence in Theorem 3). The proof that 
the intersection is already achieved for some $\nu \geqq \mu$ requires the full force of the argument of Buhštaber and Miščenko. ${ }^{2}$

2. We now turn to the first of several illustrations of the various universal coefficient theorems discussed by Adams in Lecture 1 of [1]. Highly interesting examples of Conner and Smith $[9, \S 6]$ have shown that the spectral sequence (2) can be nontrivial for finite complexes. On the other hand, we can show that the spectral sequence (2) collapses for the spectra $K(\boldsymbol{Z}), K\left(\boldsymbol{Z}_{p}\right)=K(\boldsymbol{Z}) \wedge \boldsymbol{Z}_{p}$, bu and the $\bmod p$ reductions bu $\wedge \boldsymbol{Z}_{p}$ ( $p$ is a prime). This is accomplished by first computing the $E^{2}$-terms for $K\left(\boldsymbol{Z}_{p}\right)$ and $b u \wedge \boldsymbol{Z}_{p}$ and then observing that $E^{2}=E^{\infty}$ by a comparison with the homology of $K\left(\boldsymbol{Z}_{p}\right)$ and $b u \wedge \boldsymbol{Z}_{p}$. Secondly we show by examining Bockstein's that the $E^{2}$-terms for $K(\boldsymbol{Z})$ and $b u$ have no elements of order $p^{2}$ (a known property of the homology of $K(\boldsymbol{Z})$ and $b u$ ), and then argue easily that the spectral sequence (2) also collapses in these cases.

We write $M U_{*}=M U_{*}\left(S^{0}\right)$ for the coefficient bordism ring. The computation of $\operatorname{Tor}_{*, *}^{M U_{*}}\left(M U_{*}(X), \boldsymbol{Z}\right)$ for $X=K\left(\boldsymbol{Z}_{p}\right)$ or $b u \wedge \boldsymbol{Z}_{p}$ requires a knowledge of the $M U_{*}$-modules $M U_{*}\left(K\left(\boldsymbol{Z}_{p}\right)\right) \cong H_{*}\left(M U ; \boldsymbol{Z}_{p}\right)$ and $M U_{*}\left(b u \wedge \boldsymbol{Z}_{p}\right) \cong b u_{*}(M U) \otimes \boldsymbol{Z}_{p}$ which is provided amply by R. E. Stong in [14, Chapter 7]. A free resolution of the $M U_{* \text {-module }}$ $\boldsymbol{Z}$ is provided by the Koszul resolution of the polynomial algebra $M U_{*}=Z\left[x_{1}, x_{2}, \cdots\right], \operatorname{deg} x_{i}=2 i^{3}$

3. We now comment on the relation between the reduced homology functors $M U_{*}()$ and $b u_{*}()$ on the category of based finite complexes. There is a natural transformation $[9, \S 10] \zeta: M U_{*}() \rightarrow b u_{*}()$ and the striking theorem of Conner and Smith [9, Theorem 10.6] that the factorization

$$
\tilde{\zeta}: M U_{*}(X) \otimes_{M U_{*}} b u_{*} \rightarrow b u_{*}(X)
$$

is an isomorphism if and only if hom. $\operatorname{dim} \cdot M U^{*} M U_{*}(X) \leqq 2$. We shall complement this result in two ways.

The unit $S \rightarrow b u$ of the ring spectrum $b u$ induces a natural transformation

$$
\text { Fe: } M U_{*}(X)=\pi_{*}(X \wedge M U) \rightarrow b u_{*}(X \wedge M U),
$$

the Hurewicz homomorphism. $\mathcal{F}$ admits a factorization

${ }^{2}$ An alternative treatment can be based on the result of B. Gray in Topology 5 (1966), p. 242, that for an inverse system $\left\{A_{n}\right\}$ of countable abelian groups the vanishing of proj $\lim ^{1} A_{n}$ is equivalent to the Mittag-Leffler condition.

${ }^{3} \mathrm{~A}$ detailed account of $\S / 2$ is given in On the complex bordism of Eilenberg.MacLane spaces and connective coverings of BU. I, to appear in Topology. 


$$
\tilde{F C}: M U_{*}(X) \otimes_{M U_{*}} b u_{*}(M U) \rightarrow b u_{*}(X \wedge M U)
$$

which can be identified with the external product

$$
M U_{*}(X) \otimes_{M U_{*}} M U_{*}(b u) \rightarrow M U_{*}(X \wedge b u) .
$$

Then also $\tilde{\mathfrak{H}}$ is an isomorphism if and only if hom. $\operatorname{dim} . M U_{*} M U_{*}(X) \leqq 2$.

The theorem of Stong [13] and A. Hattori [10] leads directly to the conclusion that the Hurewicz homomorphism (5) is a split monomorphism if $M U_{*}(X)$ is a free $M U_{*}$-module (i.e. if $H_{*}(X ; \boldsymbol{Z})$ has no torsion). An argument due essentially to T. tom Dieck $[15, \S 6]$ then shows that $\mathcal{H C}$ is also a monomorphism if hom. $\operatorname{dim}_{M U_{*}} M U_{*}(X) \leqq 1$. This last result and techniques of [9] now lead easily to the fact that $X$ admits $a$ "bu*-resolution" (by analogy to the $U$-bordism resolutions of $[9, \S 2])$ if and only hom. $\operatorname{dim} . M U_{*} M U_{*}(X) \leqq 2$.

4. There is a universal coefficient theorem

$$
\operatorname{Ext}_{M U_{*}}^{*, *}\left(M U_{*}(X), M U^{*}\right) \Rightarrow M U^{*}(X)
$$

as well as a version with $\boldsymbol{Z}_{p}$ coefficients:

$$
\operatorname{Ext}_{M U_{*} \otimes}^{*, *} Z_{p}\left(M U_{*}\left(X ; \boldsymbol{Z}_{p}\right), M U^{*} \otimes \boldsymbol{Z}_{p}\right) \Rightarrow M U^{*}\left(X ; \boldsymbol{Z}_{p}\right) .
$$

Applied to Eilenberg-MacLane spectra, (8) and (9) lead to the following analogues of the results of D. W. Anderson and L. Hodgkin [2] (see also [7]) on the $K$-theory of Eilenberg-MacLane spaces. Let $\pi$ be a countable abelian group and $K(\pi)$ the associated EilenbergMacLane spectrum.

THEOREM 5. If $\pi$ is a torsion group then $M U^{*}(K(\pi))=0$.

TheOREM 6. $M U^{t}(K(\pi)) \cong M U^{t-1} \otimes \operatorname{Ext}(\pi \otimes Q, Z)$.

Theorem 7. If $\pi$ is finitely generated then proj $M U^{*}(K(\pi))=0$.

The main algebraic step in the proof of Theorem 5 is the following result.

Proposition 8. Let $R$ be a polynomial algebra $k\left[x_{1}, x_{2}, \cdots\right]$ over $a$ field $k$ on an infinite sequence of indeterminates. Let $A$ be an R-module such that $\otimes_{R} A$ is an exact functor on the category of coherent $R$-modules. Then $\operatorname{Ext}_{R}^{*}(k, A)=0$. In particular $\operatorname{Ext}_{R}^{*}(k, R)=0$.

For coherence see Lecture 5 of $[1]$ or $[9, \S 1]$. The proof is in two parts. First introduce the ideal $I_{n}=\left(x_{1}, \cdots, x_{n}\right) \subset R$ and show by duality in the Koszul complex [8, Chapter VIII, Example 7] that 
$\operatorname{Ext}_{R}^{p}\left(R / I_{n}, A\right) \cong \operatorname{Tor}_{n-p}^{R}\left(R / I_{n}, A\right)$, hence $\operatorname{Ext}_{R}^{p}\left(R / I_{n}, A\right)=0$ unless $p=n$. Then justify taking an inverse limit.

\section{REFERENCES}

1. J. F. Adams, Lectures on generalized homology theories, Lecture Notes in Math., no. 99, Springer-Verlag, Berlin and New York, 1969, pp. 1-138.

2. D. W. Anderson and L. Hodgkin, The K-theory of Eilenberg-MacLane complexes, Topology 7 (1968), 317-329. MR 37 \#6924.

3. M. F. Atiyah, Characters and cohomology of finite groups, Inst. Hautes Etudes Sci. Publ. Math. No. 9 (1961), 23-64. MR 26 \#6228.

4. N. A. Baas, On the stable Adams spectral sequences, Aarhus Universitet, 1969 (preprint).

5. J. M. Boardman, Stable homotopy theory, University of Warwick, 1965 (preprint).

6. V. M. Buhštaber and A. S. Mišcenko, Elements of infinite filtration in K-theory, Dokl. Akad. Nauk SSSR 178 (1968), 1234-1237=Soviet Math. Dokl. 9 (1968), 256259.

7. - K-theory in the category of infinite cell complexes, Izv. Akad. Nauk SSSR Ser. Mat. 32 (1968), 560-604 = Math. USSR Izv. 2 (1968), 515-556.

8. H. Cartan and S. Eilenberg, Homological algebra, Princeton Univ. Press, Princeton, N. J., 1956. MR 17, 1040.

9. P. E. Conner and L. Smith, On the complex bordism of finite complexes, Inst. Hautes Etudes Sci. Publ. Math. 37 (1970).

10. A. Hattori, Integral characteristic numbers for weakly almost complex manifolds, Topology 5 (1966), 259-280.

11. J. W. Milnor, On axiomatic homology theory, Pacific J. Math. 12 (1962), 337341. MR 28 \#2544.

12. S. P. Novikov, The methods of algebraic topology from the point of view of cobordism theory, Izv. Akad. Nauk SSSR Ser. Mat. 31 (1967), 855-951= Math. USSR Izv. 1 (1967), 827-913. MR 36 \#561.

13. R. E. Stong, Relations among characteristic numbers. I, Topology 4 (1965), 267-281. MR 33 \#740.

14. - Notes on cobordism theory, Mathematical Notes, Princeton Univ. Press, Princeton, N. J., 1968.

15. T. tom Dieck, Bordism of G-manifolds and integrality theorems, Aarhus Universitet, 1968 Topology (to appear).

Yale University, New Haven, Connecticut 06520 